\title{
Prognostic model based on six PD-1 expression and immune infiltration-associated genes predicts survival in breast cancer
}

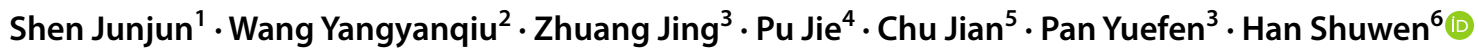

Received: 10 July 2021 / Accepted: 13 February 2022 / Published online: 1 March 2022

(c) The Author(s) 2022

\begin{abstract}
Background The prognosis of breast cancer (BC) was associated with the expression of programmed cell death-1 (PD-1). Methods BC-related expression and clinical data were downloaded from TCGA database. PD-1 expression with overall survival and clinical factors were investigated. Gene set variation analysis (GSVA) and weighted gene correlation network analysis were performed to investigate the PD-1 expression-associated KEGG pathways and genes, respectively. Immune infiltration was analyzed using the ssGSEA algorithm and DAVID, respectively. Univariate and multivariable Cox and LASSO regression analyses were performed to select prognostic genes for modeling.

Results High PD-1 expression was related to prolonged survival time $(P=0.014)$. PD-1 expression status showed correlations with age, race, and pathological subtype. ER- and PR-negative patients exhibited high PD-1 expression. The GSVA revealed that high PD-1 expression was associated with various immune-associated pathways, such as T cell/B cell receptor signaling pathway or natural killer cell-mediated cytotoxicity. The patients in the high-immune infiltration group exhibited significantly higher PD-1 expression levels. In summary, 397 genes associated with both immune infiltration and PD-1 expression were screened. Univariate analysis and LASSO regression model identified the six most valuable prognostic genes, namely IRC3, GBP2, IGJ, KLHDC7B, KLRB1, and RAC2. The prognostic model could predict survival for BC patients.

Conclusion High PD-1 expression was associated with high-immune infiltration in BC patients. Genes closely associated with PD-1, immune infiltration and survival prognosis were screened to predict prognosis.
\end{abstract}

Keywords Breast cancer · Programmed cell death-1 $\cdot$ Immune infiltration $\cdot$ Prognosis $\cdot$ Immunotherapy

Pan Yuefen and Han Shuwen contributed equally to this work.

Pan Yuefen

panyuefen663253@163.com

$\triangle$ Han Shuwen

shuwenhan985@163.com

Shen Junjun

shenjun820611@163.com

Wang Yangyanqiu

wangyangyanqiu0808@163.com

Zhuang Jing

zhuangjing0721@163.com

$\mathrm{Pu}$ Jie

pujie12072021@163.com

Chu Jian

chujian980218@163.com

1 Department of Medical Oncology, Huzhou Central Hospital, Affiliated Central Hospital Huzhou University, No. 1558, Sanhuan North Road, Wuxing District, Huzhou 313000, Zhejiang, China
2 Graduate School of Medical College of Zhejiang University, No. 268 Kaixuan Road, Jianggan District, Hangzhou 310029, Zhejiang, China

3 Department of Oncology, Huzhou Central Hospital, Affiliated Central Hospital Huzhou University, No. 1558, Sanhuan North Road, Wuxing District, Huzhou 313000, Zhejiang, China

4 Graduate School of Nursing, Huzhou University, No. 1 Bachelor Road, Huzhou 313000, Zhejiang, China

5 Graduate School of Second Clinical Medicine Faculty, Zhejiang Chinese Medical University, No. 548 Binwen Road, Binjiang District, Hangzhou 310053, Zhejiang, China

6 Department of Oncology, Huzhou Central Hospital, Affiliated Central Hospital Huzhou University, No. 1558, Sanhuan North Road, Wuxing District, Huzhou, Zhejiang, China 


$\begin{array}{ll}\text { Abbreviations } \\ \text { PD-1 } & \text { Programmed cell death-1 } \\ \text { BC } & \text { Breast cancer } \\ \text { GSVA } & \text { Gene set variation analysis } \\ \text { TNBC } & \text { Triple-negative BC } \\ \text { WGCNA } & \text { Weighted gene correlation network analysis } \\ \text { K-M } & \text { Kaplan-Meier } \\ \text { ER } & \text { Estrogen receptor } \\ \text { PR } & \text { Progesterone receptor } \\ \text { DEGs } & \text { Differentially expressed genes } \\ \text { ssGSEA } & \text { Single sample GSEA }\end{array}$

\section{Introduction}

Breast cancer (BC) is the most common malignancy in women that ranks as the main cause of cancer-related deaths in women [1]. According to the 2020 Global Cancer Statistics, BC accounted for $11.7 \%$ of the 19.3 million new cancer cases and $6.9 \%$ of the 10 million cancer-related deaths in women [2]. With the progress in early diagnosis and synthetic therapeutic strategies, $\mathrm{BC}$ prognosis improved, with a 5-year survival rate of over $80 \%$ for non-metastatic $\mathrm{BC}$ [3]. However, $20-30 \%$ of the $\mathrm{BC}$ cases demonstrate a metastatic disease following diagnosis and primary tumor treatment, with a 25\% 5-year survival rate and approximately $90 \%$ of cancer-related deaths attributed to metastasis [4]. In metastatic $\mathrm{BC}$, the curative goals are prolonging the survival and maintaining the quality of life $[5,6]$. Immunotherapy is one of the current treatment methods for malignant tumors, which is expected to bring survival benefits to $\mathrm{BC}$ patients [7].

Tumor cell immune escape is the major reason responsible for malignant tumor treatment difficulties. Immunity in the human body is mainly mediated by $\mathrm{T}$ cell-related cellular immunity, and $\mathrm{T}$ cell activation to develop the immune response depends on the second signal delivered from costimulatory molecules $[8,9]$. Programmed cell death-1 (PD1, also named PDCD1) and its ligand PD-L1 (also named CD274) belong to the CD28/B7 family, functioning as costimulatory molecules involved in the regulation of immune responses [10]. PD-1 is an inhibitory receptor induced by the activation of the immunoglobulin superfamily and is mainly expressed in T cells, whereas its ligand PD-L1 is mainly expressed in antigen-presenting cells [11]. When PD-L1 and PD-1 interact, the tumor T lymphocyte immune response is suppressed, so that tumor cells can escape the immune response [10]. Specific antibodies blocking the PD-1/PD-L1 axis could enhance the tumor $\mathrm{T}$ lymphocyte immune function and promote immune activity [12].

An increasing number of studies have shown the effect of the PD-1/PD-L1 blockade in the improvement of the adverse outcome in advanced malignant melanoma [13] and other solid tumors, such as non-small cell lung cancer [14], bladder cancer [15], or head and neck cancer [16]. BC is known for being weakly immunogenic with a lower mutational load than other tumor types, and therefore $\mathrm{BC}$, has not experienced the advances in immunotherapy yet [17]. Noske et al. demonstrated that the tumor-infiltrating lymphocyte density in $\mathrm{BC}$ showed significant correlations with PD-1 expression in the tumor cells and PD-1/PD-L1 expression in the immune cells, and PD-1-positive immune cells in triple-negative $\mathrm{BC}$ (TNBC) were related to a significantly favorable disease-free survival [18]. Recently, the PD-1/ PD-L1 blockade has also been evaluated in BC, especially in TNBC, and promising results could be observed [17]. For example, Schmid et al. suggested that in early TNBC, a higher proportion of patients showed pathological complete response among those receiving pembrolizumab (humanized monoclonal anti-PD1 antibody) combined with neoadjuvant chemotherapy (64.8\%) than those receiving placebo combined with neoadjuvant chemotherapy (51.2\%) [19]. In the KEYNOTE-355 phase III clinical trial, pembrolizumab plus chemotherapy presented a significant and clinically meaningful improvement in the progression-free survival compared to placebo plus chemotherapy among patients with metastatic TNBC with a combined positive score of $\geq 10$ [20]. These results suggest that the PD-1/PD-L1 blockade is an emerging novel therapeutic strategy for BC. However, its underlying regulatory mechanisms are still unclear.

Therefore, based on the expression and clinical data in TCGA database, we investigated PD-1 expression, prognostic value, and clinical correlations. Moreover, using weighted gene correlation network analysis (WGCNA) and the LASSO regression algorithm, PD-1 expression and tumor-infiltrating immune cell-associated biomarkers were identified to establish a prognostic risk model. Effective biomarkers and accurate prognosis are critical for healthcare decision evaluation and anti-PD-1 therapeutic response optimization. Prognostic models are reportedly available to stratify patients with different prognoses and predict overall survival. Our study illustrates novel aspects of PD-1 with potential relevance of biological regulation and immunotherapy response stratification.

\section{Methods}

\section{PD-1 expression with survival analysis and clinical factors}

The $\log 2(\mathrm{fpkm}+1)$ RNA-Seq data, as well as the clinical and survival data of BC, in TCGA database were acquired from the UCSC Genome Browser. The genes were annotated according to the human gene annotation file (version 22) provided by GENCODE. We extracted the data on PD-1 and PD-L1 
expression. PD-1 and PD-L1 expression with overall survival and PD-1 expression with clinical factors, including age, race, tumor stage, pathologic T/N/M stage, and estrogen receptor (ER), her2 receptor, and progesterone receptor (PR) status were investigated using the survival package (version 2.42-6) and ggstatsplot (version 0.6.5) in R. K-M survival analysis among BC subtypes, such as ER+/HER2-, HER2+, TNBC was performed using the survival package in $\mathrm{R}$.

\begin{tabular}{llll}
\hline & HER-2 & ER & PR \\
\hline HER-2+ & + & $-/+$ & $-/+$ \\
TNBC & - & - & - \\
ER+/HER2- & - & + & $-/+$ \\
\hline
\end{tabular}

\section{Identification of PD-1-associated genes}

GSVA analysis was conducted to calculate the enrichment score of each KEGG pathway using the GSVA software (version 1.36.2) [21]. High- and low-expression PD-1 groups were divided using the limma package (version 3.10.3) [22] in $\mathrm{R}$ according to median value. The classical Bayesian method provided by the limma package (version 3.10.3) in $\mathrm{R}$ was used to screen DEGs associated with PD-1. Weighted gene correlation network analysis (WGCNA) using the WGCNA package (version 1.61) [23] was performed to select key correlating modules with PD-1 expression and PD-1-associated genes.

\section{Identification of immune infiltration-associated genes}

Based on the single sample GSEA (ssGSEA) algorithm, the enrichment score of each immune cell in the sample was calculated using the GSVA package (version 1.36.2) in R. Unsupervised clustering was then performed using the heatmap package (version 1.0.12), to assign samples into high- and low-infiltration groups. Immune infiltration-associated genes were screened using the limma package in $\mathrm{R}$.

\section{Identification of PD-1 expression and immune infiltration-associated genes}

DAVID (version 6.8) was used to investigate the involved Gene Ontology (GO)-biological processes and KEGG pathways of overlapping genes associated with PD-1 expression and immune infiltration.

\section{Prognostic model establishment and validation}

Univariate and multivariable Cox and LASSO regression analyses were performed to select prognostic genes for modeling. GSE131769, GSE86166 (https://www.ncbi.nlm. nih.gov/geo/) and METABRIC (http://www.cbioportal.org) datasets were used to validate the prognostic model. The sample risk scores were calculated per the formula. Risk score $=\beta$ gene $1 \times$ exprgene $1+\beta$ gene $2 \times$ exprgene $2+\ldots+\beta$ genen $\times$ exprgenen. Patients were assigned into two different risk groups based on the median risk score values. The overall survival, risk score distribution, and gene expression pattern within these two risk groups were analyzed using the survminer package (version 0.4.3) in R. The distributions of Riskscores of samples grouped by clinical and demographic factors, including age, race, tumor stage, pathologic T/N/M stage, and estrogen receptor (ER), her2 receptor, and progesterone receptor (PR) status were analyzed.

\section{Statistical analysis}

$P$ value or adjusted $P$ value $<0.05$ was considered as statistically significant in the whole study.

The detailed methods were presented in Methods supplement.

\section{Results}

\section{PD-1 is prognosis-related in BC}

In TCGA dataset, the PD-1 and PD-L1 prognostic values were evaluated using the survival analysis. As shown in Fig. 1A, patients with high PD-1 tended to exhibit better survival rates than those with low PD-1 $(P=0.014)$, while patients with high PD-L1 tended to exhibit no significant difference in survival rates than those with low PD-L1 $(P=0.14)$

\section{PD-1 expression with clinical factors}

To investigate the associations between the PD-1 expression and clinical factors, the patients were divided into subgroups based on different clinical factors, followed by the comparison of differences with the PD-1 expression. The results showed that the PD-1 expression exhibited no difference between the sub-groups divided by tumor stage, pathologic T/N/M, and her2 receptor status. PD-1 expression was associated with age, race, and PR and ER status (Fig. 1B). Patients aged $<60$ years had high PD-1 expression in comparison with those aged $\geq 60$ years $(P=0.020)$. Black or African American patients had high PD-1 expression in comparison with Asian patients $(P<0.001)$. ER-negative and PR-negative patients had higher PD-1 expression than those with ER-positive and PR-positive status $(P<0.01)$. 
Fig. 1 PD-1 expression association with survival and clinical factors. A The $\mathrm{K}-\mathrm{M}$ survival curves show the prognostic value of PD-1 and PD-L1 expression for breast cancer patients. B The violin plots show the differences between PD-1 expression status and age, race, tumor stage, pathologic $\mathrm{T} / \mathrm{N} / \mathrm{M}$, and estrogen receptor, her2 receptor, and progesterone receptor status. $\mathbf{C}$ The $\mathrm{K}-\mathrm{M}$ survival curves show the prognostic value of PD-1 expression among three breast cancer subtypes

A
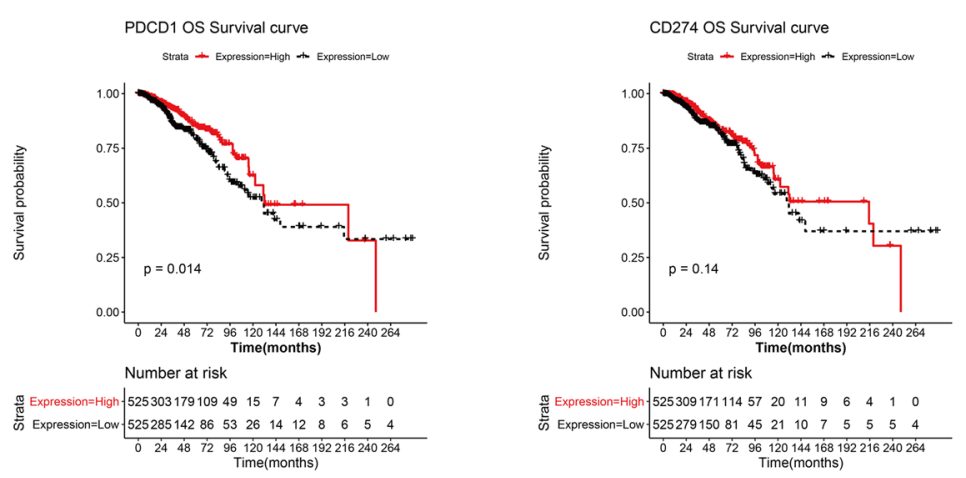

B

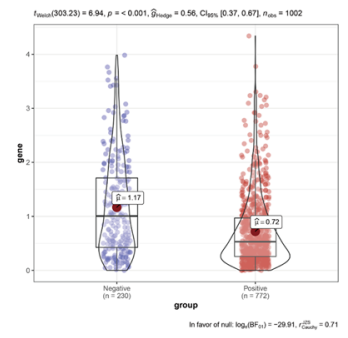

estrogen receptor status

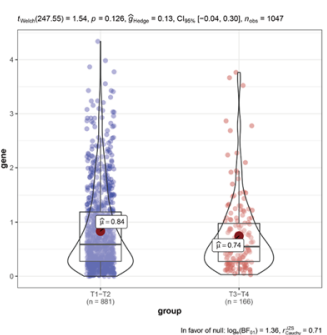

pathologic $T$

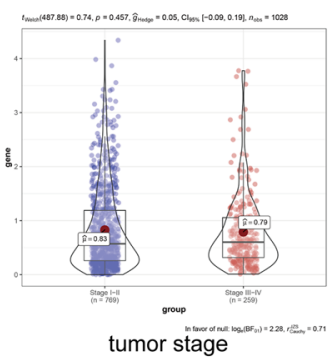

C

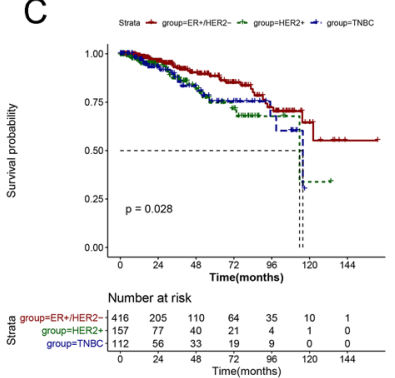

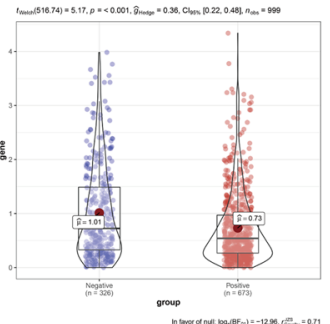

progesterone receptor status

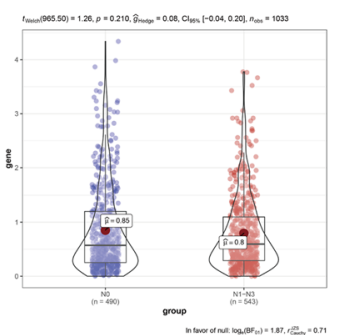

pathologic $\mathrm{N}$

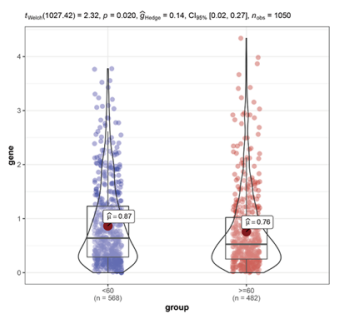

age

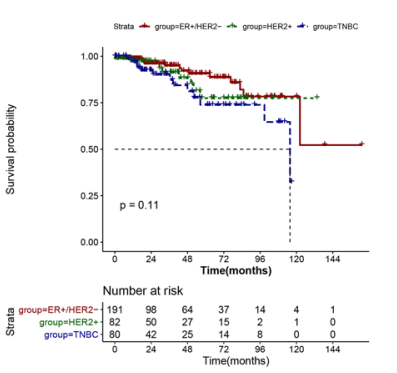

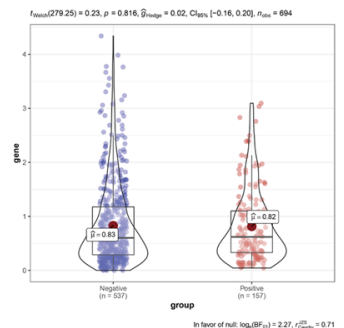

her-2 receptor status

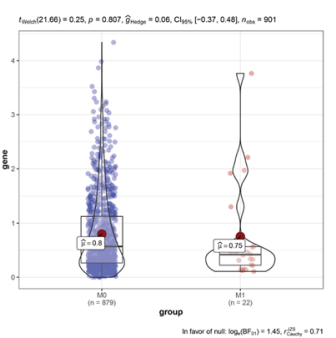

pathologic M

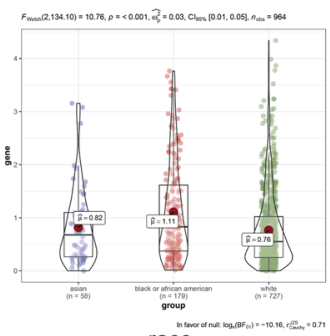

race

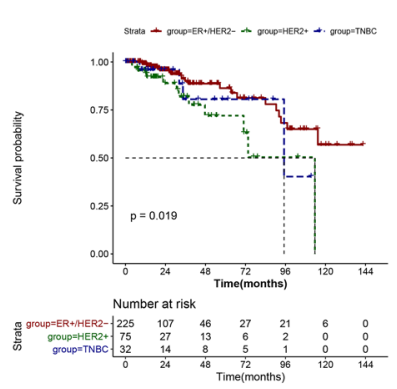




\section{Survival analysis of three BC subtypes}

As shown in Fig. 1C, there was no significant difference in the survival of three BC subtypes in high PD-1 expression group $(P=0.11)$, while there was significant difference in the survival of three BC subtypes in low PD-1 expression $(P=0.019)$. And the prognosis of HER $2+$ subtype was poor. Among all samples, there were significant differences in survival among three $\mathrm{BC}$ subtypes $(P=0.028)$. TNBC showed the worst prognosis in high PD-1 expression group, while HER2+ showed poor prognosis in low PD-1 expression group. ER+/HER2 - type has a better prognosis in both high and low PD-1 expression groups.

\section{PD-1 expression-associated pathways}

GSVA was used to investigate the differences in the KEGG pathways between the high- and low-PD-1 expression groups. We screened a total of 119 pathways showing significant differences. Figure 2 displays the top 20 pathways in these two expression groups. Multiple immune-associated pathways were enriched in the high PD-1 expression group, such as B cell and T cell receptor signaling pathways, natural killer cell-mediated cytotoxicity, or chemokine signaling pathway (Fig. 2A). Various biosynthesis- and metabolism-associated pathways were visibly enriched in the low-PD-1 expression group, including nitrogen, riboflavin, steroid, butanoate, ascorbate, and aldarate metabolism, as well as steroid, valine, leucine, and isoleucine biosynthesis (Fig. 2B).

\section{PD-1 expression-associated genes}

A total of 2379 DEGs were screened between the highand low-PD-1 expression groups, and we observed that the majority of the genes ( $n=1861 ; 78.2 \%$ vs. $n=518 ; 21.8 \%$ ) were up-regulated in the high PD-1 expression group (Fig. 3A, Table S1). These 2379 DEGs were analyzed by

A

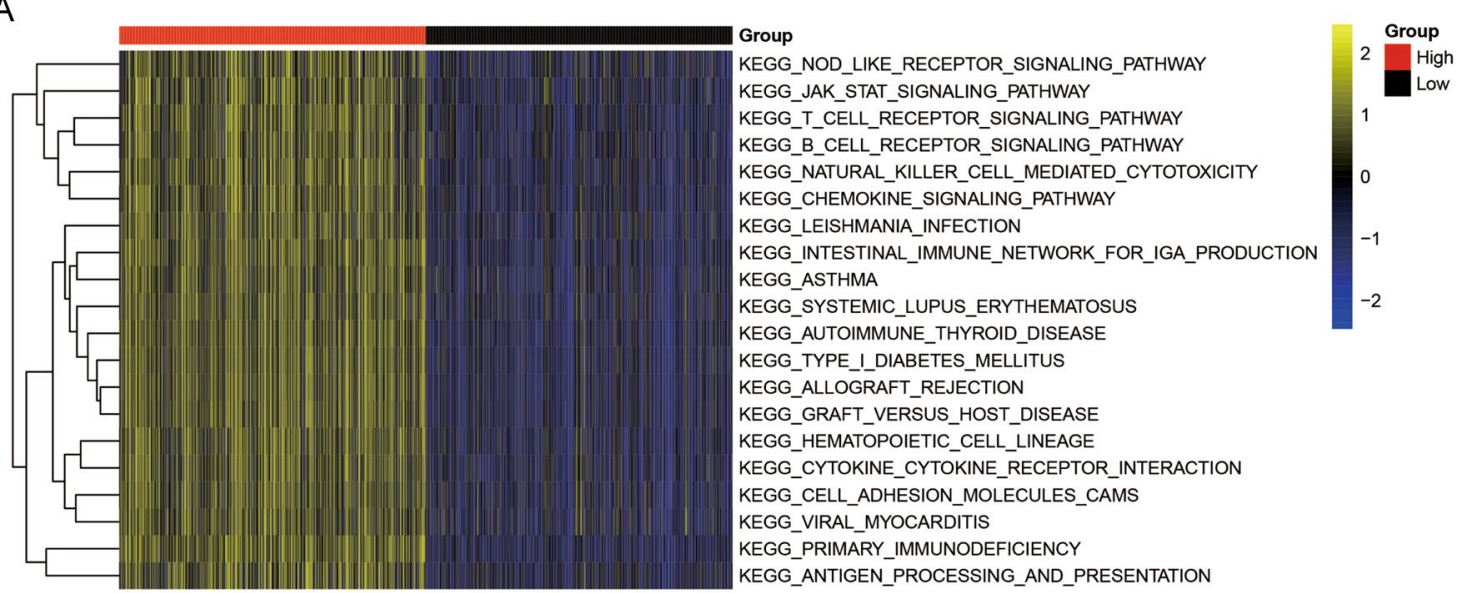

B

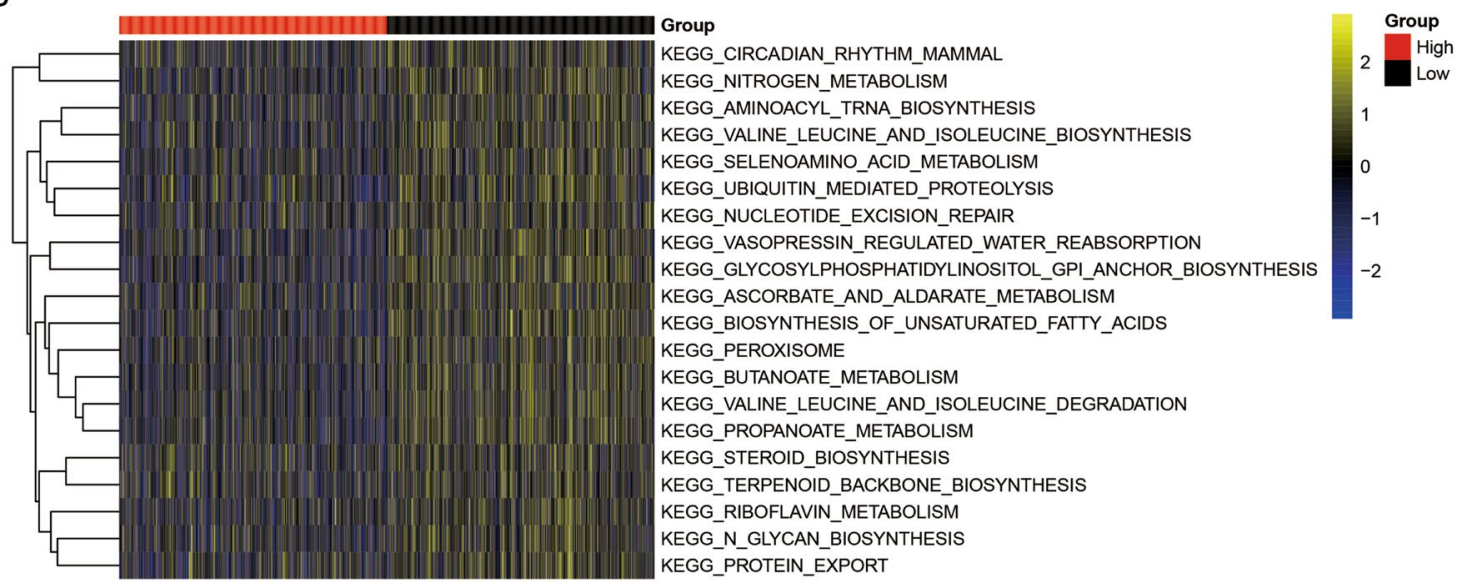

Fig. 2 Results of gene set variation analysis. The heatmaps show the significantly enriched KEGG pathways in the (A) high- and (B) low-PD-1 expression groups 


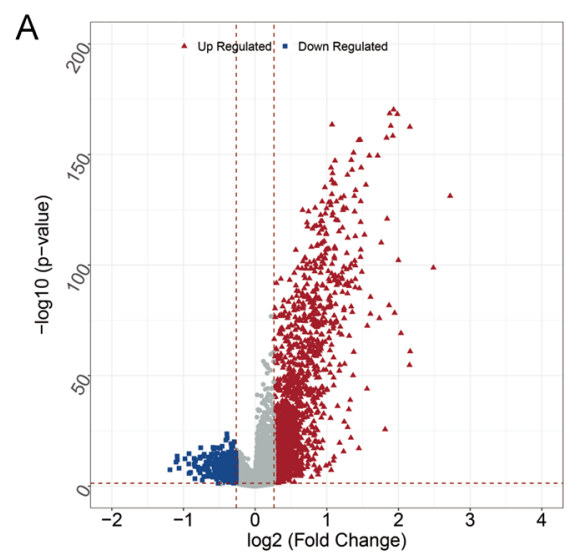

C

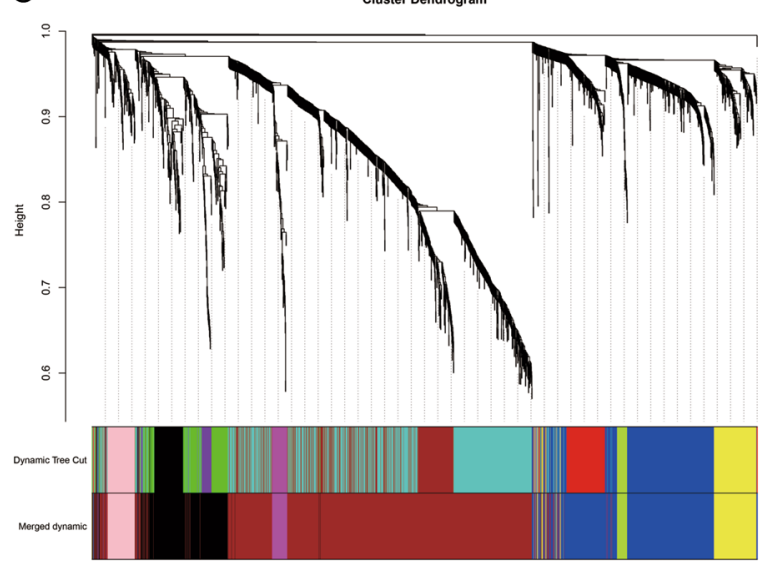

B

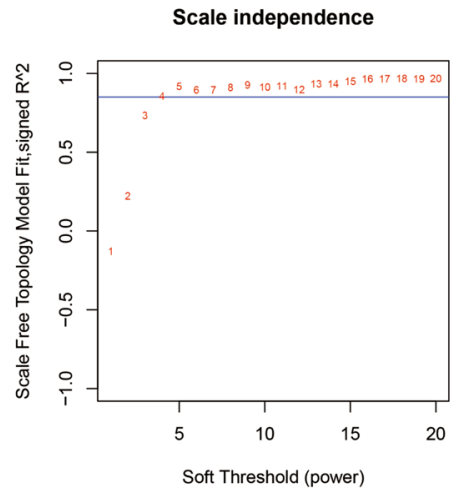

Mean connectivity

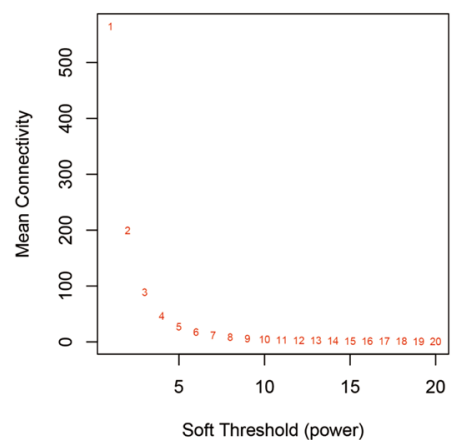

D

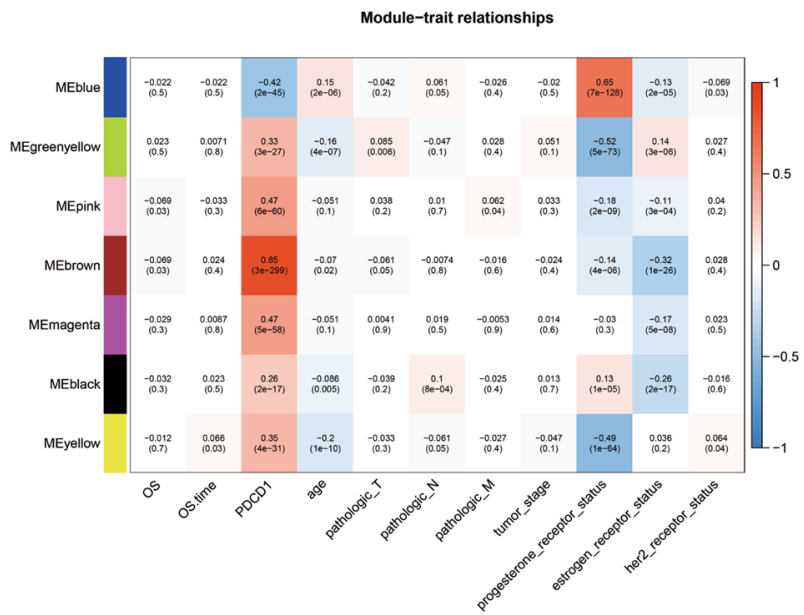

soft-threshold (power) in weighted gene correlation network analysis (WGCNA). C Cluster dendrogram generated by hierarchical clustering based on gene dissimilarity measures. D Correlations analysis for gene modules and traits

ssGSEA. Next, the samples were assigned into high- and low-infiltration groups (Fig. 4A). Samples with high PD-1 expression were mainly clustered in the high-infiltration group, and most samples with PD-1 low expression were clustered in the low-infiltration group. The high- and low-infiltration groups showed significantly different proportions of patients with high PD-1 expression (95 vs. $41 \%$ ) and patients with low PD-1 expression (5 vs. 59\%) (Fig. 4B). The patients in the high-infiltration group exhibited a significantly higher PD-1 expression level (Fig. 4C).

In addition, 429 DEGs were screened between the highand low-immune infiltration groups (Table S2). These 429 genes were considered immune infiltration-associated genes. Most genes $(n=392,91.4 \%)$ were up-regulated in the high-infiltration group.

\section{Identification of immune infiltration-associated genes}

The 23 immune cell infiltration abundance cases were evaluated using the enrichment score calculated by 
A

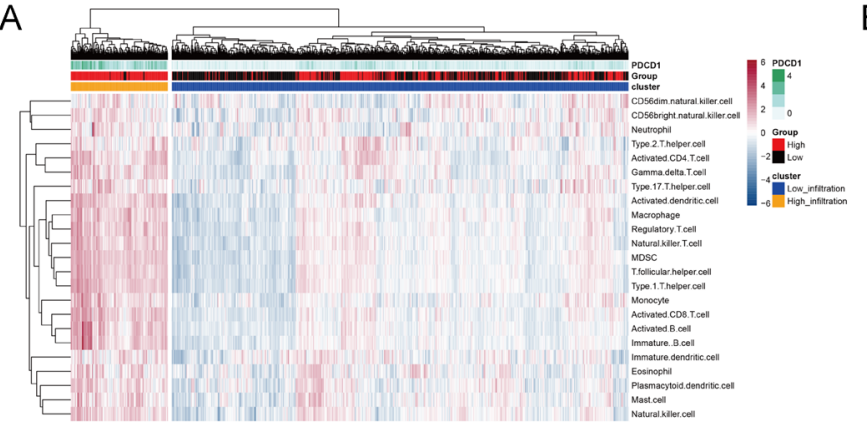

B

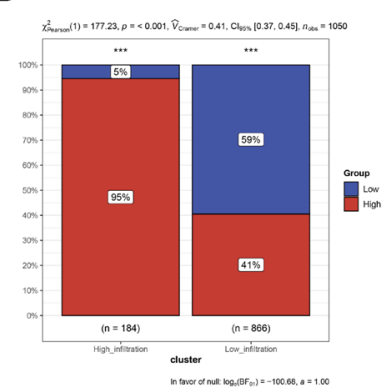

C

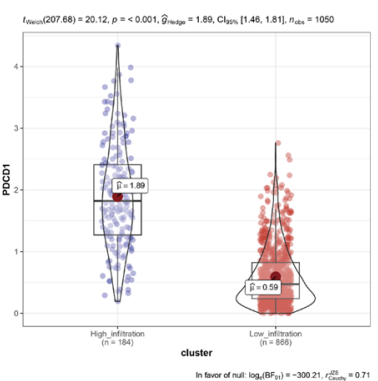

D

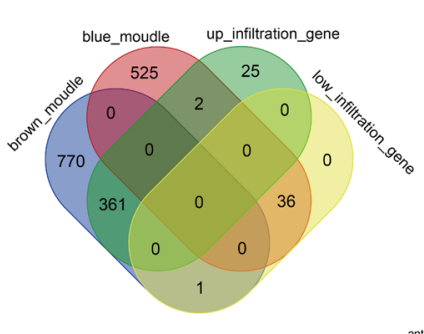

E

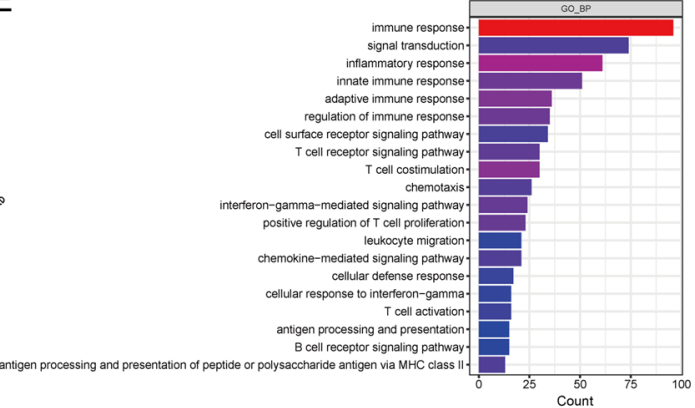

$\mathrm{F}$

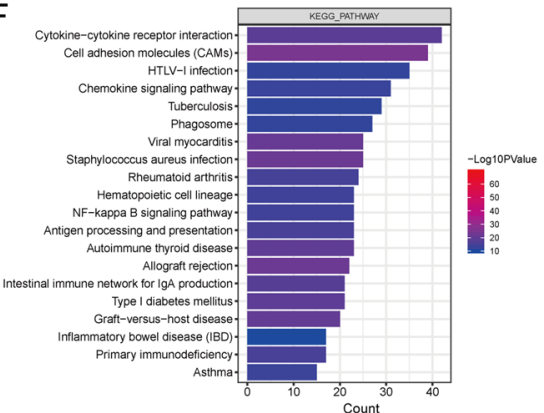

Fig. 4 Immune infiltration- and PD-1 expression-associated genes. A Heatmap showing the enrichment score of 23 immune cell infiltration cases. B Histogram shows the proportion of patients with high- and low-PD-1 expression status in different infiltration groups. C Violin plots showing the PD-1 expression status differences between the high- and low-infiltration groups. D Venn plot showing the overlap- ping genes between the PD-1 expression-associated genes (genes in two WGCNA modules) and immune infiltration-associated genes (differentially expressed genes in the high- and low-infiltration groups). E Top 20 significantly enriched GO-biological processes and F KEGG pathways

\section{Immune infiltration- and PD-1 expression-associated genes}

The PD-1 expression- (screened by WGCNA) and immune infiltration-associated genes were merged to identify genes associated with both immune infiltration and PD-1 expression. The Venn analysis resulted in 397 overlapping genes, comprising 361 up-regulated and 36 down-regulated genes (Fig. 4D, Table S3).

Next, we carried out a functional enrichment analysis for these overlapping genes. We found a significant enrichment of a total of $254 \mathrm{GO}$-biological processes and 45 KEGG pathways. Figure 4E-F shows the top 20 GObiological processes and pathways, indicating that these genes were mainly implicated in immune-related biological processes, containing immune, inflammatory, innate immune, and adaptive immune responses, as well as T cell co-stimulation, proliferation, or activation. The top 20 pathways included cytokine-cytokine receptor interaction, chemokine signaling pathway, cell adhesion molecules, and antigen processing and presentation.

\section{Prognostic model}

Among the 397 immune infiltration- and PD-1 expression-associated genes, our univariate Cox regression analysis identified 70 genes correlating with prognosis $(P<0.01)$. Based on the optimal parameter lambda. min, the LASSO regression model was used to select the most valuable prognostic genes from these 70 genes. As a result, six genes were selected, namely IRC3, GBP2, IGJ, KLHDC7B, KLRB1, and RAC2 (Fig. 5A). Next, we performed multivariable Cox regression analysis for the calculation of the prognostic correlation coefficient for these six genes (Table S3). Based on the prognostic correlation coefficient and the expression value, we established a sixgene prognostic model. After calculating the risk score for each sample, we assigned them all into two different risk groups according to the median risk score. The expression of these six genes gradually decreased with a risk score from low to high (Fig. 5B). Patients in the high-risk group were associated with adverse survival compared to those in the low-risk group (Fig. 5C). 
A

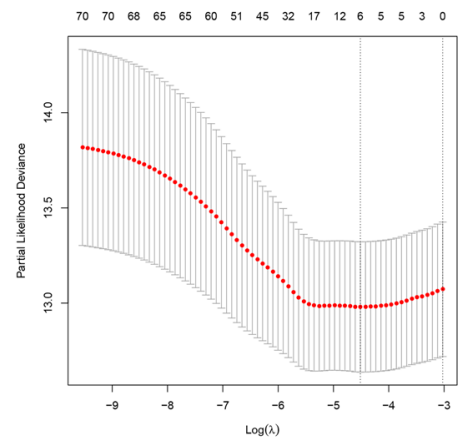

B
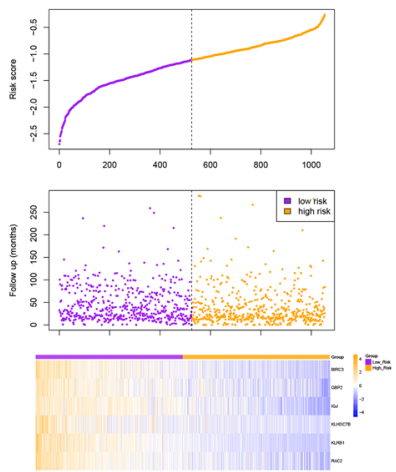

C

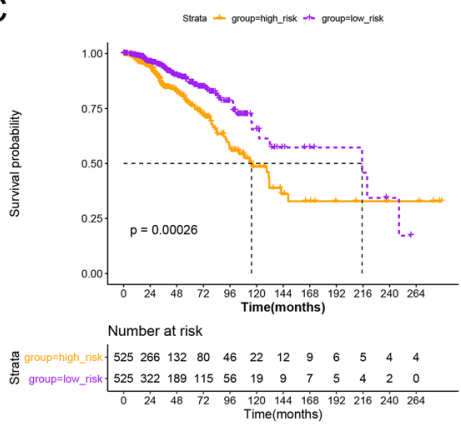

D
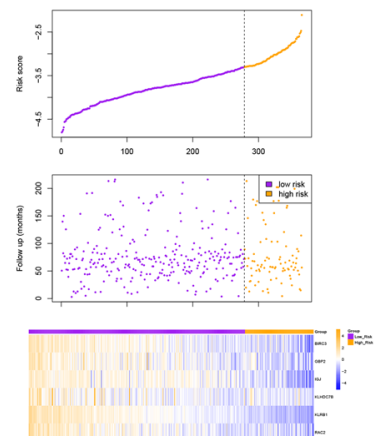

GSE86166
$\mathrm{F}$
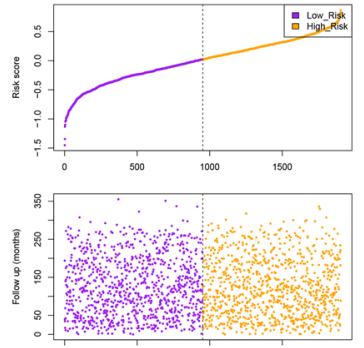

METABRIC
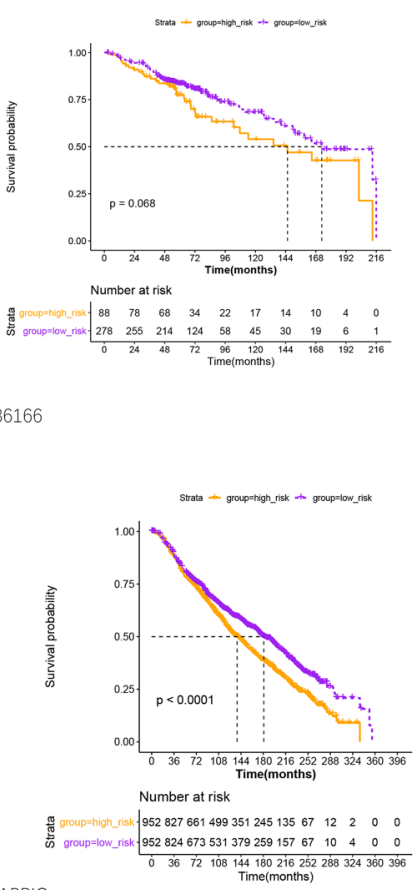

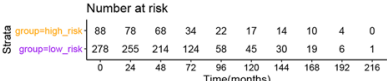

Fig. 5 Prognostic model establishment and validation. A Selection of the optimal parameter lambda.min in the LASSO regression model. The dotted curve on the left and the right indicate lambda.min and lambda.1se, respectively. The model constructed based on lambda.1se is the simplest as a small number of genes were involved. The model constructed based on lambda.min is more accurate, involving a larger number of genes. For the horizontal axis, the upper numbers represent the numbers of variables corresponding to different $\lambda$ values at bottom. The vertical axis represents partial likelihood deviance. B Risk score distribution between high- and low-risk groups. The risk score can assign patients into high- and low-risk groups (upper). The survival status of patients in the high- and low-risk groups. The verti-

\section{External validation of the prognostic model}

The GSE131769, GSE86166 and METABRIC datasets for the external validation of the prognostic model. Similarly,
E

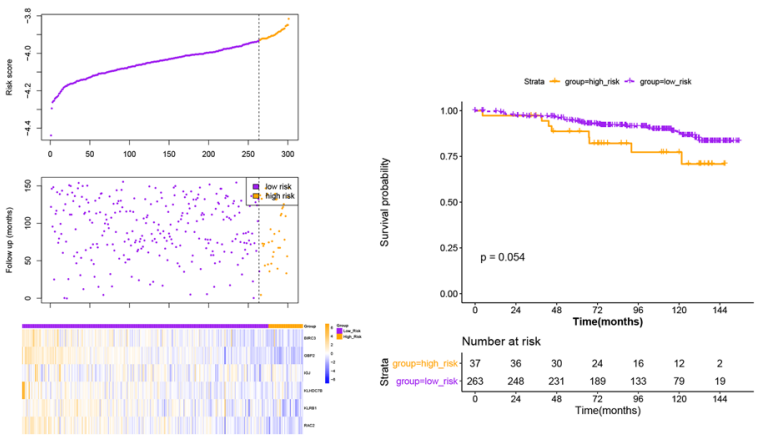

GSE131769
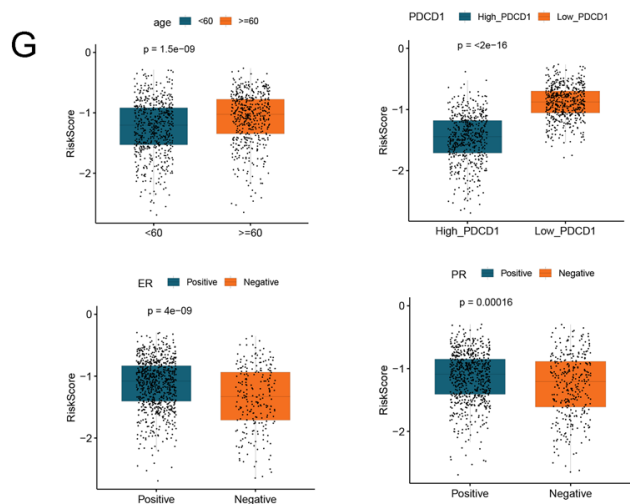

cal and horizontal axes represent the follow-up (months) and the risk score (middle), respectively. Heatmaps of gene expression patterns with risk scores from low to high. The vertical and horizontal axes represent the six genes and the risk score, respectively. The expression of these six genes gradually decreased with the risk score from low to high (bottom). C K-M curves showing the overall survival between the high- and low-risk groups. D Prognostic model validation in the GSE86166 dataset. E Prognostic model validation in the GSE131769 dataset. F Prognostic model validation in the METABRIC dataset. G The association between clinical parameters (age, PD1 expression, ER and PR status) and risk scores were analyzed

the risk score calculated using the six-gene prognostic model could classify the patients into two risk groups. The expression of these six genes gradually decreased with risk scores from low to high. Moreover, the patients in the high-risk 
group tended to exhibit worse survival compared to those in the low-risk group. The survival differences showed no statistical significance, with $P=0.054, P=0.068$ in the GSE131769 and GSE86166 (Fig. 5D-E), respectively. However, the survival differences showed statistical significance, with $P<0.0001$ in METABRIC datasets (Fig. 5F). There were significant differences in RiskScore between underand over-60-year groups $(P<0.01)$, PDCD1 high- and lowexpression groups $(P<0.01)$, ER-positive and -negative groups $(P<0.01)$, and PR-positive and -negative groups $(P=0.00016)$ (Fig. 5G). There were no significant differences in different race, tumor stage, pathologic T/N/M stage, and her2 receptor groups $(P>0.05)$.

\section{Discussion}

PD-1/PD-L1 are important co-stimulatory molecules involved in the regulation of immune responses. When PD-L1 and PD-1 interact, the immune response of tumor T lymphocytes is suppressed, so that tumor cells can escape the immune response [10]. The anti-PD-1/PD-L1 antibodies have been approved by the FDA in an unprecedentedly fast manner to treat various cancers. Recently, the evaluation of the PD-1/PD-L1 blockade in BC is ongoing, especially in TNBC, showing promising results [17]. Therefore, we investigated the potential underlying PD-1/PD-L1 regulatory mechanisms and their prognostic value in $\mathrm{BC}$.

Using the data in TCGA, the association between PD-1/ PD-L1 expression status, patient survival, and PD-1/PD-L1 expression status with clinical factors was investigated. Patients with high PD-1 expression demonstrated a favorable prognosis compared to those with low PD-1 expression $(P=0.014)$, while patients with high and low PD-L1 expression showed no significant difference in survival time. Moreover, Matikas et al. [24], Jiang et al. [25] suggested that the expression of PD-1 protein correlated with improved overall survival for BC patients. These results suggested that high PD-1 expression might be a favorable prognostic marker in BC. However, we found that ER- and PR-negative patients had high PD-1 expression compared with patients displaying ER- and PR-positive status $(P<0.01)$. Similar results were also found in a previous study [25], which indicated that ER-negative ( 4.35 vs. $1.82, P<0.001)$ and PR-negative (3.47 vs. $1.86, P<0.001) \mathrm{BC}$ patients had higher PD-1 expression than that in ER-positive and PR-positive patients. This result suggested that PD-1 expression varies among the different BC subtypes and might explain why PD-1/PD-L1 blockade showed promising results, especially in TNBC. Meanwhile, patients who aged $<60$ years had high PD-1 expression in comparison with those aged $\geq 60$ years $(P=0.020)$. Black or African American patients had high PD-1 expression in comparison with Asian patients $(P<0.001)$. It indicated that age and race should be considered when using PD1 therapy.

To investigate the potential underlying mechanism of survival difference between high and low PD-1 expression, GSVA and immune infiltration analysis were performed. GSVA revealed that various immune-associated pathways were enriched in the high PD-1 expression group, such as $\mathrm{B}$ cell and $\mathrm{T}$ cell receptor signaling pathways, natural killer cell-mediated cytotoxicity, or chemokine signaling pathway. Moreover, the immune infiltration analysis revealed that patients in the high-infiltration group showed a significantly elevated PD-1 expression level. These results suggested that the improved survival of patients with higher PD-1 expression levels might be mainly attributed to increased immune infiltration and the activation of immune response-associated genes.

There were 1861 up-regulated DEGs and 518 down-regulated DEGs between high and low PD1 expression groups. WGCNA analysis of these DEGs showed that positive and negative PD1 expression were significantly correlated with age, PR and ER status. It indicated that age and BC subtypes should be taken into full consideration when using PD1 therapy again. Later, 429 DEGs were found between the high- and low- immune infiltration groups. Then, 397 genes associated with both immune infiltration and PD-1 expression were screened and 70 genes of among them correlated with the prognosis. These genes were mainly implicated in immune-related biological processes, suggesting that prognosis correlated with immune response. LASSO regression model was used to select the most valuable prognostic genes, identifying six genes, namely IRC3, GBP2, IGJ, KLHDC7B, KLRB1, and RAC2. IRC3 is involved in mitochondrial genome stability maintenance [26]. Genomic instability is a hallmark of tumorigenesis, mainly associated with the accumulation of DNA damage [27]. The dysregulation of IRC3 expression might affect DNA replication and repair in BC. GBP2 encodes a guanylate-binding protein induced by interferon- $\gamma$ [28]. Increased GBP2 expression in $\mathrm{BC}$ showed correlations with improved survival and tumor-infiltrating $T$ cell [29]. GBP2 could suppress cell metastasis and mitochondrial fission in BC cells both in vitro and in vivo [30]. High IGJ expression showed correlations with improved overall early $\mathrm{BC}$ patient survival [31]. Tumors with high proportion of Ki67-positive cells and ductal tumors exhibited the highest KLHDC7B expression, suggesting that KLHDC7B could be a biomarker for poorly differentiated BC [32]. KLHDC7B may inhibit BC tumorigenesis by regulating interferon signals [33]. KLRB1 is expressed by NK cells and encodes the NK cell CD161 receptor, involving regulation of NK cell function [34]. NK cells are lymphocytes that modulate cytotoxicity and secrete cytokines following immune stimulation. KLRB1 expression was inhibited in tumor tissues, and it potentiated be 
a prognostic biomarker in carcinoma. RAC2 is expressed solely in hematopoietic cells that play an important role in neutrophils and lymphocytes [35]. High RAC2 expression was associated with adverse survival, suggesting its potential as a promising prognostic marker [36].

Based on the 6 genes, Riskscore model was constructed. External datasets, including GSE131769, GSE86166 and METABRIC were used for detection the Riskscore. There was no significant difference in survival between the highand low-risk groups in GSE131769 and GSE86166 datasets. However, there was significant difference in survival between the high- and low-risk groups in METABRIC, and the high-risk group had worse prognosis. The difference in verification results between GSE131769, GSE86166 databases and METABRIC database is probably due to the insufficient sample size in GSE131769 and GSE86166 databases. The validation result of METABRIC database that owns large sample size is more accurate and convincing. Immune response played important role in the anti-tumor process. These six immune response-associated genes correlated with $\mathrm{T}$ cells, NK cells, interferon, etc. Up-regulated or downregulated expressions of these six genes might affect the function of immune cells and secretion of immune-related molecules. So the prognostic model established based on these six immune response-associated genes could predict overall $\mathrm{BC}$ patient survival, suggesting their prognostic value in $\mathrm{BC}$.

In conclusion, we investigated PD-1 expression status with patient survival and clinical factors in BC. High PD-1 expression could predict a favorable $\mathrm{BC}$ prognosis. $\mathrm{PD}-1$ expression levels varied among different subtypes, with high PD-1 expression in ER- and PR-negative patients. The favorable patient survival with high PD-1 expression might be mainly attributed to the increased immune infiltration and activation of immune response-associated genes. We identified six genes, namely IRC3, GBP2, IGJ, KLHDC7B, KLRB1, and RAC2, associated with both immune infiltration and PD-1 expression as key prognostic genes in BC. The prognostic model based on these six genes could predict overall BC patient survival. These genes might be potential prognostic biomarkers to predict the response to anti-PD-1 therapy.

Supplementary Information The online version contains supplementary material available at https://doi.org/10.1007/s12282-022-01344-2.

Acknowledgements The authors gratefully acknowledge the database available to us for this study.

Author contributions All authors participated in the conception and design of the study; conceptualization: HS and PY; methodology: WY, $\mathrm{PJ}$ and CJ; formal analysis and investigation: SJ, PY, and CJ; writingoriginal draft preparation: $\mathrm{SJ}$ and $\mathrm{ZJ}$; writing - review and editing: WY; funding acquisition: SJ; supervision: HS. All authors read and approved the paper.
Funding This work was supported by the Public Welfare Technology Application Research Program of Huzhou (No. 2019GY17) and Health Technology Projects of Zhejiang Province (No. 2022KY1219).

Data availability The datasets generated during the current study are not publicly available but obtained from corresponding authors on reasonable request.

\section{Declarations}

Conflict of interest The authors declare that no conflicts of interest exist.

Open Access This article is licensed under a Creative Commons Attribution 4.0 International License, which permits use, sharing, adaptation, distribution and reproduction in any medium or format, as long as you give appropriate credit to the original author(s) and the source, provide a link to the Creative Commons licence, and indicate if changes were made. The images or other third party material in this article are included in the article's Creative Commons licence, unless indicated otherwise in a credit line to the material. If material is not included in the article's Creative Commons licence and your intended use is not permitted by statutory regulation or exceeds the permitted use, you will need to obtain permission directly from the copyright holder. To view a copy of this licence, visit http://creativecommons.org/licenses/by/4.0/.

\section{References}

1. Britt KLCJ, Phillips KA. Key steps for effective breast cancer prevention. Nat Rev Cancer. 2020;20:417-36.

2. Sung H, Ferlay J, Siegel RL, Laversanne M, Soerjomataram I, Jemal A, Bray F. Global cancer statistics 2020: GLOBOCAN estimates of incidence and mortality worldwide for 36 cancers in 185 countries. CA Cancer J Clin. 2021;71:209-49.

3. Allemani C, Matsuda T, Di Carlo V, Harewood R, Matz M, Nikšić M, Bonaventure A, Valkov M, Johnson CJ, Estève J, Ogunbiyi OJ, Azevedo ESG, Chen WQ, Eser S, Engholm G, Stiller CA, Monnereau A, Woods RR, Visser O, Lim GH, Aitken J, Weir HK, Coleman MP. individual records for 37513025 patients diagnosed with one of 18 cancers from 322 population-based registries in 71 countries. Lancet. 2018;391:1023-75.

4. Liang Y, Zhang H, Song X, Yang Q. Metastatic heterogeneity of breast cancer: molecular mechanism and potential therapeutic targets. Semin Cancer Biol. 2020;60:14-27.

5. Harbeck N, Gnant M. Breast cancer. Lancet. 2017;389:1134-50.

6. Barzaman K, Karami J, Zarei Z, et al. Breast cancer: Biology, biomarkers, and treatments. Int Immunopharmacol. 2020;84:106535.

7. Emens LA. Breast cancer immunotherapy: facts and hopes. Clin Cancer Res. 2018;24:511-20.

8. Schorer M, Kuchroo VK, Joller N. Role of co-stimulatory molecules in T helper cell differentiation. Adv Exp Med Biol. 2019;1189:153-77.

9. Azuma M. Co-signal molecules in T-cell activation: historical overview and perspective. Adv Exp Med Biol. 2019;1189:3-23.

10. Fife BT, Bluestone JA. Control of peripheral T-cell tolerance and autoimmunity via the CTLA-4 and PD-1 pathways. Immunol Rev. 2008;224:166-82.

11. Han Y, Liu D, Li L. PD-1/PD-L1 pathway: current researches in cancer. Am J Cancer Res. 2020;10:727-42.

12. Jiang $\mathrm{Y}, \mathrm{Zhao} \mathrm{X}, \mathrm{Fu}$ J, Wang H. Progress and challenges in precise treatment of tumors with PD-1/PD-L1 blockade. Front Immunol. 2020;11:339. 
13. Mahoney KM, Freeman GJ, McDermott DF. The next immunecheckpoint inhibitors: PD-1/PD-L1 blockade in melanoma. Clin Ther. 2015;37:764-82.

14. Xia L, Liu Y, Wang Y. PD-1/PD-L1 blockade therapy in advanced non-small-cell lung cancer: current status and future directions. Oncologist. 2019;24:S31-41.

15. Inman BA, Longo TA, Ramalingam S, Harrison MR. Atezolizumab: a PD-L1-blocking antibody for bladder cancer. Clin Cancer Res. 2017;23:1886-90.

16. Kok VC. Current understanding of the mechanisms underlying immune evasion from PD-1/PD-L1 immune checkpoint blockade in head and neck cancer. Front Oncol. 2020;10:268.

17. Planes-Laine G, Rochigneux P, Bertucci F, Chrétien AS, Viens P, Sabatier R, Gonçalves A. PD-1/PD-L1 targeting in breast cancer: the first clinical evidences are emerging. A literature review. Cancers (Basel). 2019;11(7):1033.

18. Noske A, Möbus V, Weber K, Schmatloch S, Weichert W, Köhne CH, Solbach C, Ingold Heppner B, Steiger K, Müller V, Fasching P, Karn T, van Mackelenbergh M, Marmé F, Schmitt WD, Schem C, Stickeler E, Loibl S, Denkert C. Relevance of tumourinfiltrating lymphocytes, PD-1 and PD-L1 in patients with highrisk, nodal-metastasised breast cancer of the German adjuvant intergroup node-positive study. Eur J Cancer. 2019;114:76-88.

19. Schmid PCJ, Pusztai L, McArthur H, Kümmel S, Bergh J, Denkert C, Park YH, Hui R, Harbeck N, Takahashi M, Foukakis T, Fasching PA, Cardoso F, Untch M, Jia L, Karantza V, Zhao J, Aktan G, Dent R, O'Shaughnessy J, KEYNOTE-522 Investigators. Pembrolizumab for early triple-negative breast cancer. N Engl J Med. 2020;382:810-21.

20. Cortes J, Cescon DW, Rugo HS, Nowecki Z, Im SA, Yusof MM, Gallardo C, Lipatov O, Barrios CH, Holgado E, Iwata H, Masuda N, Otero MT, Gokmen E, Loi S, Guo Z, Zhao J, Aktan G, Karantza V, Schmid P. Pembrolizumab plus chemotherapy versus placebo plus chemotherapy for previously untreated locally recurrent inoperable or metastatic triple-negative breast cancer (KEYNOTE-355): a randomised, placebo-controlled, doubleblind, phase 3 clinical trial. Lancet. 2020;396:1817-28.

21. Hänzelmann S, Castelo R, Guinney J. GSVA: gene set variation analysis for microarray and RNA-seq data. BMC Bioinformatics. 2013;14:7

22. Smyth GK. limma: linear models for microarray data. New York: Springer; 2005.

23. Langfelder P, Horvath S. WGCNA: an R package for weighted correlation network analysis. BMC Bioinformatics. 2008;9:559.

24. Matikas A, Zerdes I, Lövrot J, Sifakis E, Richard F, Sotiriou C, Rassidakis G, Bergh J, Valachis A, Foukakis T. PD-1 protein and gene expression as prognostic factors in early breast cancer. ESMO Open. 2020;5:e001032.

25. Jiang C, Cao S, Li N, Jiang L, Sun T. PD-1 and PD-L1 correlated gene expression profiles and their association with clinical outcomes of breast cancer. Cancer Cell Int. 2019;19:233.
26. Sedman T, Gaidutšik I, Villemson K, Hou Y, Sedman J. Double-stranded DNA-dependent ATPase Irc3p is directly involved in mitochondrial genome maintenance. Nucleic Acids Res. 2014;42:13214-27.

27. O'Connor MJ. Targeting the DNA damage response in cancer. Mol Cell. 2015;60:547-60.

28. Guimarães DP, Oliveira IM, de Moraes E, Paiva GR, Souza DM, Barnas C, Olmedo DB, Pinto CE, Faria PA, De Moura Gallo $\mathrm{CV}$, Small IA, Ferreira CG, Hainaut P. Interferon-inducible guanylate binding protein (GBP)-2: a novel p53-regulated tumor marker in esophageal squamous cell carcinomas. Int J Cancer. 2009;124:272-9.

29. Godoy P, Cadenas C, Hellwig B, Marchan R, Stewart J, Reif R, Lohr M, Gehrmann M, Rahnenführer J, Schmidt M, Hengstler JG. Interferon-inducible guanylate binding protein (GBP2) is associated with better prognosis in breast cancer and indicates an efficient T cell response. Breast Cancer. 2014;21:491-9.

30. Zhang J, Zhang Y, Wu W, Wang F, Liu X, Shui G, Nie C. Guanylate-binding protein 2 regulates Drp1-mediated mitochondrial fission to suppress breast cancer cell invasion. Cell Death Dis. 2017;8:e3151.

31. Larsson CEA, Winslow S, Leandersson K, Klintman M, Dahl L, Vallon-Christersson J, Häkkinen J, Hegardt C, Manjer J, Saal L, Rydén L, Malmberg M, Borg A, Loman N. Prognostic implications of the expression levels of different immunoglobulin heavy chain-encoding RNAs in early breast cancer. NPJ Breast Cancer. 2020;6:28.

32. Martín-Pardillos A, Cajal SRY. Characterization of Kelch domaincontaining protein 7B in breast tumours and breast cancer cell lines. Oncol Lett. 2019;18:2853-60.

33. Jeong G, Bae H, Jeong D, Ham J, Park S, Kim HW, Kang HS, Kim SJ. A Kelch domain-containing KLHDC7B and a long non-coding RNA ST8SIA6-AS1 act oppositely on breast cancer cell proliferation via the interferon signaling pathway. Sci Rep. 2018;8:12922.

34. Gilles SR, Yohe SL, Linden MA, Dolan M, Hirsch B, Grzywacz B. CD161 is expressed in a subset of T-cell prolymphocytic leukemia cases and is useful for disease follow-up. Am J Clin Pathol. 2019;152:471-8.

35. Lougaris V, Baronio M, Gazzurelli L, Benvenuto A, Plebani A. RAC2 and primary human immune deficiencies. J Leukoc Biol. 2020;108:687-96.

36. Liu Y, Cheng G, Song Z, Xu T, Ruan H, Cao Q, Wang K, Bao L, Liu J, Zhou L, Liu D, Yang H, Chen K, Zhang X. RAC2 acts as a prognostic biomarker and promotes the progression of clear cell renal cell carcinoma. Int J Oncol. 2019;55:645-56.

Publisher's Note Springer Nature remains neutral with regard to jurisdictional claims in published maps and institutional affiliations. 